\section{(2) OPEN ACCESS}

\title{
Pulmonary fibrosis 4 months after COVID-19 is associated with severity of illness and blood leucocyte telomere length
}

\author{
Claire F McGroder, ${ }^{1}$ David Zhang, ${ }^{1}$ Mohammad A Choudhury, ${ }_{1}^{1}$ Mary M Salvatore, ${ }^{2}$ \\ Belinda M D'Souza, ${ }^{2}$ Eric A Hoffman, ${ }^{3}$ Ying Wei, ${ }^{4}$ Matthew R Baldwin (1) , \\ Christine Kim Garcia (1) 1,5
}

\begin{abstract}
- Additional supplemental material is published online only. To view, please visit the journal online (http://dx.doi. org/10.1136/thoraxjnl-2021 217031)
\end{abstract}

'Medicine/PACC, Columbia University Irving Medical Center, New York, New York, USA ${ }^{2}$ Radiology, Columbia University Medical Center, New York, New York, USA

${ }^{3}$ Radiology, Internal Medicine, and Biomedical Engineering,

University of lowa Carver

College of Medicine, lowa City, Iowa, USA

${ }^{4}$ Biostatistics, Columbia University Medical Center, New York, New York, USA Institute of Genomic Medicine, Center for Precision Medicine and Genomics, Columbia University Irving Medical Center, New York, NY, USA

\section{Correspondence to}

Dr Christine Kim Garcia, Pulmonary and Critical Care Medicine, Columbia University Irving Medical Center, New York, NY 10032-3784, USA ckg2116@cumc.columbia.edu

MRB and CKG are joint senior authors.

Received 5 February 2021 Revised 6 April 2021 Accepted 13 April 2021 Published Online First 29 April 2021

Check for updates

(c) Author(s) (or their employer(s)) 2021. Re-use permitted under CC BY-NC. No commercial re-use. See rights and permissions. Published by BMJ.

To cite: McGroder CF,

Zhang D, Choudhury MA,

et al. Thorax

2021:76:1242-1245.

\section{ABSTRACT}

The risk factors for development of fibrotic-like radiographic abnormalities after severe COVID-19 are incompletely described and the extent to which CT findings correlate with symptoms and physical function after hospitalisation remains unclear. At 4 months after hospitalisation, fibrotic-like patterns were more common in those who underwent mechanical ventilation (72\%) than in those who did not (20\%). We demonstrate that severity of initial illness, duration of mechanical ventilation, lactate dehydrogenase on admission and leucocyte telomere length are independent risk factors for fibrotic-like radiographic abnormalities. These fibroticlike changes correlate with lung function, cough and measures of frailty, but not with dyspnoea.

\section{INTRODUCTION}

Reports of hospitalised COVID-19 survivors show that there are persistent symptoms, radiographic abnormalities and physiological impairments months after the initial illness. ${ }^{1} 2$ Persistent chest imaging abnormalities and histopathological findings of lung fibrosis were also found in a majority of survivors of the SARS-CoV-1 2003 outbreak, ${ }^{34}$ suggesting that the SARS viruses may lead to a worse fibroproliferative response than other pneumonias.

Cohort studies of COVID-19 survivors report that severity of the initial illness is associated with a greater risk of persistent CT abnormalities, ${ }^{1256}$ especially for patients requiring supplemental oxygen or mechanical ventilation, but independent clinical, biomarker and genomic risk factors have not been identified. Also, the extent to which CT findings correlate with symptoms and physical function remains unclear. To address knowledge gaps, we conducted a prospective cohort study of survivors hospitalised with severe COVID-19, half of whom were mechanically ventilated, with 4-month follow-up. We sought to characterise associations of pulmonary radiographic and physiologic sequela of severe COVID-19, and to identify independent risk factors for the development of post-COVID fibrosis.

\section{METHODS}

Additional details are included in the supplemental materials.

We conducted a single-centre prospective cohort study of adults hospitalised between 1 March 2020 and 15 May 2020 who required supplemental oxygen. At 4 months after hospitalisation, participants underwent a non-contrast high-resolution chest CT (HRCT) scan, pulmonary function testing, measurement of 6-minute walk distance (6MWD), assessment of the frailty phenotype and a blood draw for isolation of genomic DNA. Radiographic patterns were categorised and quantitated using a severity scoring system developed by ARDSnet and used in acute respiratory distress syndrome (ARDS) survivors, ${ }^{7}$ and classified into two groups (nonfibrotic or fibrotic). Fibrotic-like patterns included those with reticulations, traction bronchiectasis or honeycombing. Telomere length of genomic DNA isolated from blood drawn at the 4-month follow-up visit was measured by a quantitative PCR assay. $^{8}$

We calculated Spearman's rank correlation coefficients between continuous data. We created separate generalised additive logistic models (GAMs) to test adjusted associations between the risk of fibrotic-like patterns on CT scan and independent continuous variables identified in univariable analysis. Due to the moderate cohort size and rate of fibrotic-like radiographic abnormalities, we used generalised covariate balanced propensity scores to adjust for potential confounders. We estimated adjusted ORs using logistic regression models if there was no evidence of non-linearity.

\section{RESULTS}

We enrolled 76 patients meeting eligibility criteria (online supplemental figure S1); demographic and clinical features are shown in online supplemental table S1. All participants required supplemental oxygen during hospitalisation, and 32 (42\%) required mechanical ventilation.

A median of 4.4 (IQR 4.0-4.8) months after hospitalisation, the most common radiographic abnormality was ground glass opacities (43\%), followed by reticulations (39\%) and traction bronchiectasis (28\%) (figure 1, online supplemental table S2). Fibroticlike patterns were more common in those who were mechanically ventilated compared with those who were not $(72 \%$ vs $20 \%, \mathrm{p}=0.001)$ (online supplemental tables S1 and S4). In unadjusted analyses, those with fibrotic-like patterns were significantly more likely to be male, have shorter telomeres, higher admission Sequential Organ Failure Assessment (SOFA) scores, higher lactate dehydrogenase (LDH) levels and have received steroids or anti-interleukin-6 
A

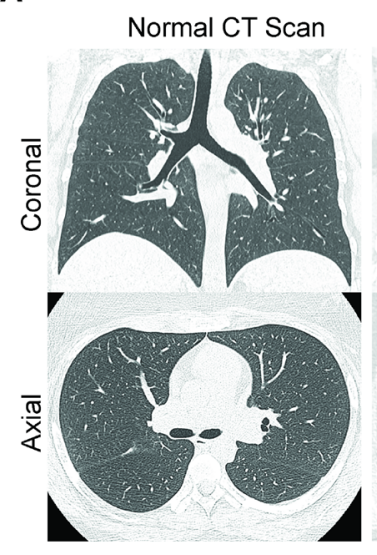

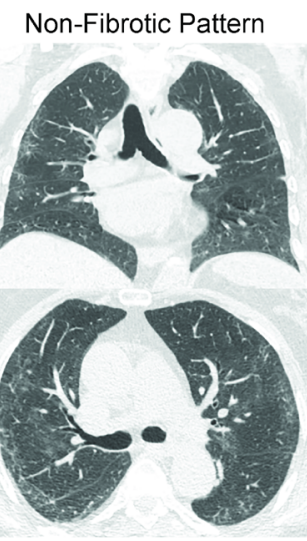

GGO Score: 5.6

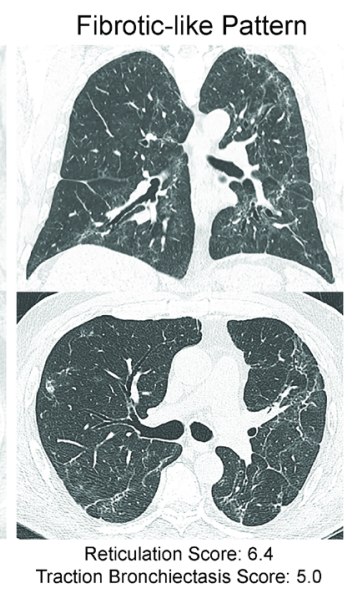

B

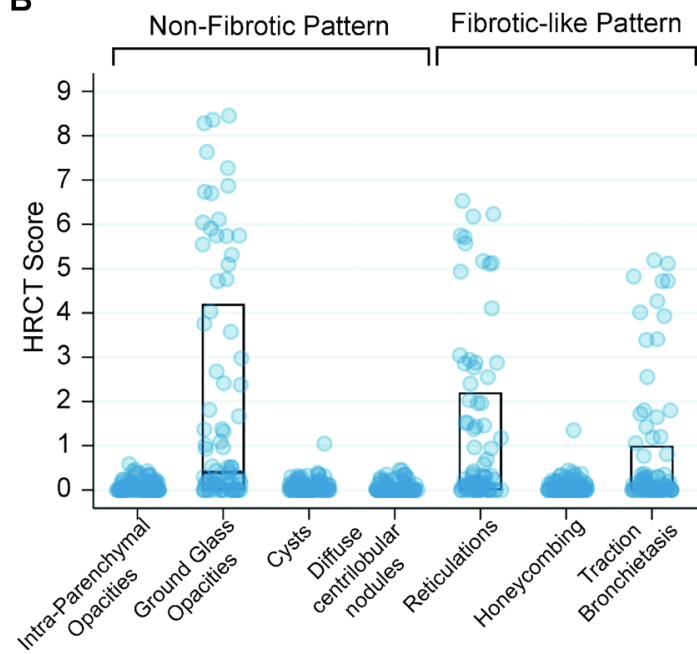

Figure 1 High-resolution CT (HRCT) scans of the chest from COVID-19 survivors. (A) Representative CT chest scans demonstrating no abnormalities (left), non-fibrotic patterns (middle) and fibrotic-like patterns (right). The upper panels show a coronal section and the lower panels show an axial image at the level just below the carina. The scan with a non-fibrotic pattern had a ground glass opacities (GGO) score of 5.6 (84th percentile in the group). The CT scan with a fibrotic-like pattern had a reticulation score of 6.4 (98th percentile), a traction bronchiectasis score of 5.0 (95th percentile) and no honeycombing. (B) Chest HRCT scores for radiographic patterns observed in the study cohort. The middle line of the boxplot represents the median score; bottom and top lines represent the 25th and 75th percentile, respectively. Where no lines are seen, the 25th, 50th and 75th percentile scores were all 0 . The extent of each pattern was graded using a scoring system developed by ARDSnet. ${ }^{7}$ The possible range of scores was $0-20$ from all categories of abnormalities, except traction bronchiectasis, which had a possible range of 0-5.

receptor blockade (online supplemental table S3). Qualitative measures of non-fibrotic and fibrotic patterns as scored by radiologists were closely associated with quantitative scores ascertained by adaptive multiple feature method (AMFM) texture analysis (online supplemental figure S2).

Participants had an array of functional deficits (online supplemental table S5). Overall, 40 (53\%) had a reduced diffusion capacity, $78 \%$ had a decreased $6 \mathrm{MWD}, 18 \%$ remained $>10 \%$ below baseline weight and 53\% had weak grip strength.

Ground glass, reticulations and traction bronchiectasis scores correlated more strongly with reduction in diffusion capacity $(\rho-0.34,-0.64$ and -0.49 , respectively, all $\mathrm{p}<0.01)$ than FVC (table 1). Ground glass correlated with the frailty phenotype score, while reticulation and traction bronchiectasis correlated

Table 1 Spearman correlation coefficients of radiographic and dyspnoea scores with pulmonary function, 6-minute walk distance, frailty and symptoms

\begin{tabular}{|c|c|c|c|c|c|c|c|c|c|}
\hline \multirow[b]{2}{*}{ CT pattern } & \multicolumn{3}{|c|}{ DLCO (\% predicted) } & \multicolumn{3}{|c|}{ FVC $(\%$ predicted $)$} & \multicolumn{3}{|c|}{ 6MWD (m) } \\
\hline & R2 & $r$ & $P$ value & R2 & $r$ & $P$ value & R2 & $r$ & $\begin{array}{l}P \\
\text { value }\end{array}$ \\
\hline Ground glass opacities & 0.12 & -0.34 & $0.003^{*}$ & 0.06 & -0.25 & $0.03^{*}$ & 0 & -0.02 & 0.92 \\
\hline Reticulations & 0.41 & -0.64 & $<0.001^{*}$ & 0.04 & -0.21 & 0.07 & 0 & -0.02 & 0.8 \\
\hline \multirow[t]{2}{*}{ Traction bronchiectasis } & 0.24 & -0.49 & $<0.001^{*}$ & 0.05 & -0.23 & $0.04^{*}$ & 0 & -0.05 & 0.69 \\
\hline & \multicolumn{3}{|c|}{ Frailty phenotype score } & \multicolumn{3}{|c|}{ Cough scale } & \multicolumn{3}{|c|}{ UCSD SOBQ } \\
\hline CT pattern & R2 & $r$ & $P$ value & R2 & $r$ & $P$ value & R2 & $r$ & $\begin{array}{l}P \\
\text { value }\end{array}$ \\
\hline Ground glass opacities & 0.21 & 0.46 & $<0.001^{*}$ & 0 & 0.07 & 0.56 & 0.02 & 0.14 & 0.23 \\
\hline Reticulations & 0.05 & 0.23 & $0.04^{*}$ & 0.07 & 0.26 & $0.02^{*}$ & 0 & 0.05 & 0.66 \\
\hline \multirow[t]{3}{*}{ Traction bronchiectasis } & 0.03 & 0.16 & 0.17 & 0.06 & 0.25 & $0.03^{*}$ & 0 & 0.07 & 0.57 \\
\hline & \multicolumn{3}{|c|}{ DLCO (\% predicted) } & \multicolumn{3}{|c|}{ FVC (\% predicted) } & \multicolumn{3}{|c|}{ 6MWD (m) } \\
\hline & R2 & $r$ & $P$ value & R2 & $r$ & $P$ value & R2 & $r$ & $\begin{array}{l}P \\
\text { value }\end{array}$ \\
\hline \multirow[t]{3}{*}{ UCSD SOBQ } & 0.02 & -0.14 & 0.24 & 0.06 & -0.25 & $0.04^{*}$ & 0.06 & -0.25 & $0.03^{*}$ \\
\hline & \multicolumn{3}{|c|}{ Frailty phenotype score } & \multicolumn{3}{|c|}{ Grip strength (kg) } & \multicolumn{3}{|c|}{ Gait speed (m/s) } \\
\hline & $\mathbf{R}^{2}$ & $r$ & $P$ value & $\mathrm{R}^{2}$ & $r$ & $P$ value & $\mathrm{R}^{2}$ & $r$ & $\begin{array}{l}P \\
\text { value }\end{array}$ \\
\hline UCSD SOBQ & 0.22 & 0.47 & $<0.001^{*}$ & 0.14 & -0.37 & $0.001^{*}$ & 0.05 & -0.21 & 0.06 \\
\hline
\end{tabular}

*Significant after controlling for false discovery using the Benjamini-Hochberg method at a false discovery rate of 0.10 .

DLCO, diffusion capacity for carbon monoxide; 6MWD, 6-minute walk distance; UCSD SOBQ, University of California San Diego Shortness of Breath Questionnaire. 

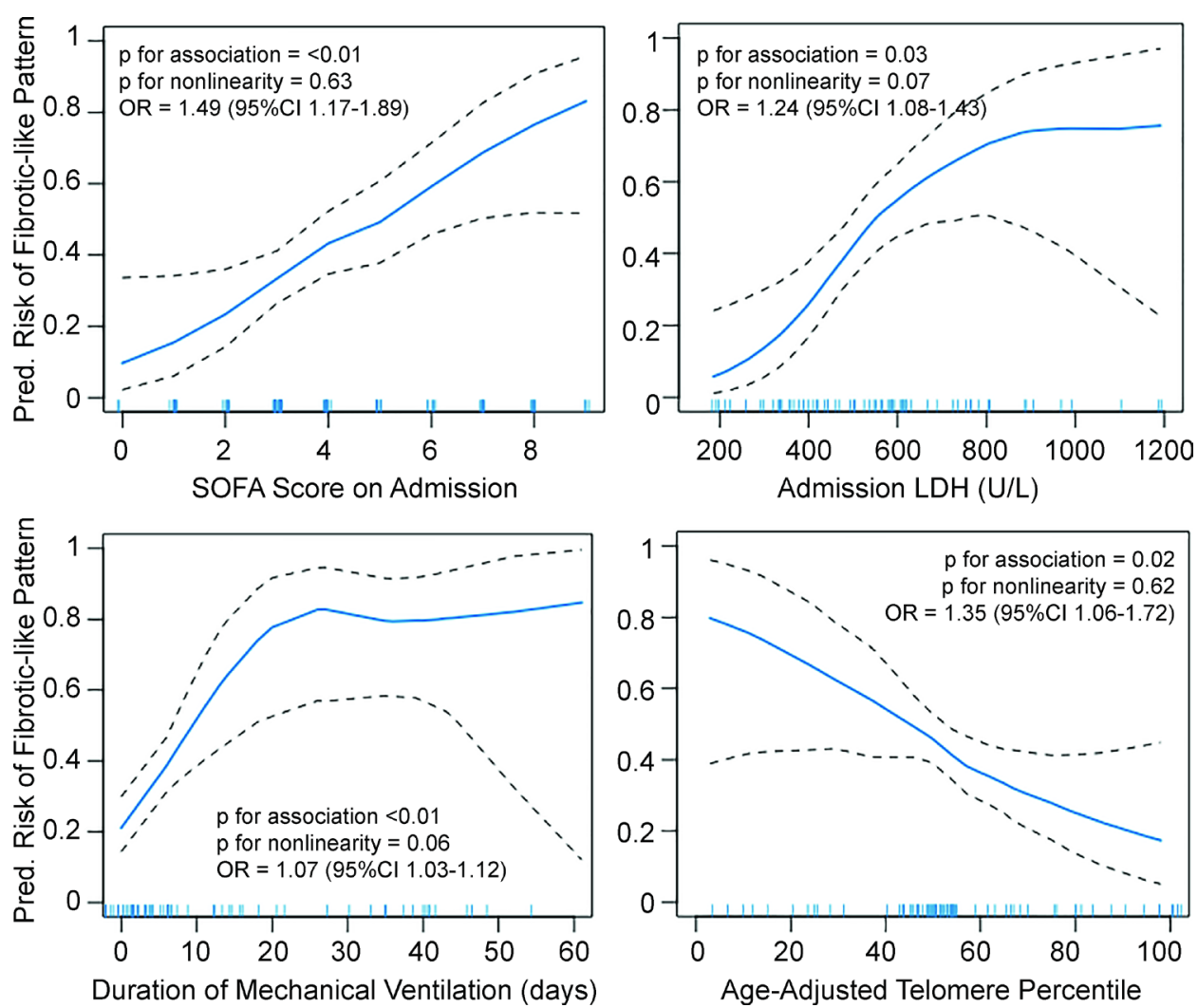

Figure 2 Continuous associations of fibrotic-like patterns with admission Sequential Organ Failure Assessment (SOFA) score (top left), lactate dehydrogenase (LDH) levels (top right), duration of mechanical ventilation in days (bottom left) and age-adjusted leucocyte telomere length percentile (bottom right) using generalised additive models with locally weighted smoothing (LOESS). Blue line represents predicted values. Black dashed lines are $95 \% \mathrm{Cls}$. Hash marks along the $\mathrm{x}$-axis indicate individual study participants. Since there was no evidence for non-linearity in the generalised additive models, we estimated adjusted ORs using logistic regression models. All models are adjusted for a common set of potential confounders (age, sex, race/ethnicity, days since infection, body mass index, pack-years of smoking, treatment with steroids while hospitalised) and the other independent variables of interest (SOFA score, LDH, days of mechanical ventilation, telomere length). OR for SOFA score is per point increase, for LDH is per 50-point increase, for mechanical ventilation is per day increase, and for telomere length is per $10 \%$ decrease in percent-predicted value.

with cough. Dyspnoea correlated more strongly with markers of weakness and deconditioning, including increased frailty score and reduced grip strength, than radiographic abnormalities (table 1). Similarly, the 6MWD was associated with the dyspnoea score and not with radiographic abnormalities.

Fully adjusted GAMs showed that both admission SOFA score and percent-predicted telomere length were linearly associated with the predicted risk of fibrotic-like radiographic abnormalities (figure 2). Duration of mechanical ventilation varied linearly with the predicted risk of fibrotic-like patterns through 20 days and plateaued with more prolonged mechanical ventilation. LDH levels also plateaued at higher levels. In fully adjusted logistic regression models, every 1-point increase in SOFA score, 50 -point increase in LDH and 1 ventilator-day was associated with 1.49 (95\% CI 1.17 to 1.89 ), 1.24 (95\% CI 1.08 to 1.43 ) and 1.07 (95\% CI 1.03 to 1.12 ) higher odds of fibrotic-like patterns on CT scan, respectively. Each $10 \%$ decrease in ageadjusted telomere length was associated with a 1.35 higher odds of fibrotic-like patterns (95\% CI 1.06 to 1.72 ). Sensitivity analyses are shown in online supplemental figures S3 and S4.

\section{DISCUSSION}

Pulmonary fibrosis is a feared complication of respiratory infections. We found that among survivors of severe COVID-19, 20\% of non-mechanically ventilated and $72 \%$ of mechanically ventilated individuals had fibrotic-like radiographic abnormalities
4 months after hospitalisation. The presence of these radiographic abnormalities correlates with decrements in lung function, cough and frailty. Greater initial severity of illness, longer duration of mechanical ventilation and shorter blood leucocyte telomere length are independent risk factors for the development of fibrotic-like abnormalities.

Fibrosis was measured in this study both subjectively, in a manner congruent with other population-based, ${ }^{9}$ ARDS, ${ }^{7}$ SARSCoV- $1^{4}$ and COVID-19 ${ }^{12610}$ studies, as well as objectively using texture analysis. ${ }^{11}$ We include reticulations as a manifestation of fibrotic-like patterns to facilitate comparison to prior postinfectious studies, ${ }^{7}$ including post-acute COVID-19 studies, ${ }^{2} 10$ and acknowledge that reticulations may either resolve or progress over time. ${ }^{4}$ The presence of the pulmonary function degradations associated with these radiographic findings at 4 months is concerning for potential long-term damage. In the absence of longer-term follow-up, it is unclear if these functional and radiographic abnormalities represent permanent lung scarring.

This is the first study to identify age-adjusted telomere length as an independent risk factor for post-COVID lung fibrosis. Short blood leucocyte telomere lengths have been shown to be a risk factor for the development of different subtypes of fibrotic interstitial lung disease, including idiopathic pulmonary fibrosis (IPF). ${ }^{12}$ Here, we also find that longer telomere lengths appear to be protective, thus, this genomic biomarker may measure the balance of profibrotic and antifibrotic susceptibilities. 
Limitations of this study include its small size, the lack of replication cohort and the need to use a propensity score to adjust for covariates. It is possible that acute illness may affect telomere length ${ }^{13}$ or that some imaging abnormalities were pre-existing. Patients were hospitalised prior to US Food and Drug Administration (FDA)-approved therapies, yet half received steroids.

Still, this study reveals significant respiratory symptoms and morbidity associated with severe COVID-19. Dyspnoea, reported by many survivors, ${ }^{14}$ correlates more strongly with muscle strength and frailty measures than radiographic pattern scores, suggesting persistent extrapulmonary effects, including cardiovascular or neuromuscular dysfunction. Additional prospective studies are needed to characterise temporal changes of post-COVID-19 fibrotic abnormalities, and clinical trials are needed to investigate therapeutic options to promote its resolution.

\section{Twitter Claire F McGroder @Claire_McGroder}

Acknowledgements The authors wish to thank the patients for their participation, Deanna D. Rivas and Mason W. Amelotte for excellent technical assistance, Junfeng Guo, PhD, for assistance with the AMFM analysis, and the Safety Monitoring Board (SMB) Chair Joao De Andrade, MD, and SMB members Anil Vachani, MD, and Anna Rozenshtein, MD. The study sponsors had no role in data analysis and interpretation, manuscript writing or dissemination of results.

Contributors CM, MRB and CKG conceptualised the study. CM, MAC and MRB recruited patients and collected samples and clinical data. DZ performed experiments and analysed data. MS, BD and EAH analysed imaging studies. CM, DZ, YW, MRB and CKG performed statistical analysis. CM, MRB and CKG wrote the manuscript.

Funding This work was supported by the NIH (R01HL103676, R01HL093096 to CKG; T32HL105323 to CM; UL1TR001873 to MRB) and the Department of Defense (PR202907 to CKG and MRB).

Competing interests None declared.

Patient consent for publication Not required.

Provenance and peer review Not commissioned; externally peer reviewed.

Open access This is an open access article distributed in accordance with the Creative Commons Attribution Non Commercial (CC BY-NC 4.0) license, which permits others to distribute, remix, adapt, build upon this work non-commercially, and license their derivative works on different terms, provided the original work is properly cited, appropriate credit is given, any changes made indicated, and the use is non-commercial. See: http://creativecommons.org/licenses/by-nc/4.0/.

\section{ORCID iDs}

Matthew R Baldwin http://orcid.org/0000-0003-4670-3433

Christine Kim Garcia http://orcid.org/0000-0002-0771-1249

\section{REFERENCES}

1 Zhao Y-M, Shang Y-M, Song W-B, et al. Follow-Up study of the pulmonary function and related physiological characteristics of COVID-19 survivors three months after recovery. EClinicalMedicine 2020;25:100463.

2 Shah AS, Wong AW, Hague CJ, et al. A prospective study of 12-week respiratory outcomes in COVID-19-related hospitalisations. Thorax 2021;76:402-4.

3 Hui DS, Wong KT, Ko FW, et al. The 1-year impact of severe acute respiratory syndrome on pulmonary function, exercise capacity, and quality of life in a cohort of survivors. Chest 2005:128:2247-61.

4 Wong K-tak, Antonio GE, Hui DSC, et al. Severe acute respiratory syndrome: thinsection computed tomography features, temporal changes, and clinicoradiologic correlation during the convalescent period. J Comput Assist Tomogr 2004;28:790-5.

5 Huang $Y$, Tan C, Wu J, et al. Impact of coronavirus disease 2019 on pulmonary function in early convalescence phase. Respir Res 2020;21:163.

6 Huang W, Wu Q, Chen Z. The potential indicators for pulmonary fibrosis in survivors of severe COVID-19. J Infect 2020.

7 Burnham EL, Hyzy RC, Paine R, et al. Chest CT features are associated with poorer quality of life in acute lung injury Survivors*. Crit Care Med 2013:41:445-56.

8 Stuart BD, Lee JS, Kozlitina J, et al. Effect of telomere length on survival in patients with idiopathic pulmonary fibrosis: an observational cohort study with independent validation. Lancet Respir Med 2014;2:557-65.

9 Putman RK, Gudmundsson G, Axelsson GT, et al. Imaging patterns are associated with interstitial lung abnormality progression and mortality. Am J Respir Crit Care Med 2019:200:175-83

10 Han X, Fan Y, Alwalid O, et al. Six-Month follow-up chest CT findings after severe COVID-19 pneumonia. Radiology 2021;299:E177-86.

11 Salisbury ML, Lynch DA, van Beek EJR, et al. Idiopathic pulmonary fibrosis: the association between the adaptive multiple features method and fibrosis outcomes. Am J Respir Crit Care Med 2017:195:921-9.

12 Cronkhite JT, Xing C, Raghu G, et al. Telomere shortening in familial and sporadic pulmonary fibrosis. Am J Respir Crit Care Med 2008;178:729-37.

13 Zribi B, Uziel O, Lahav $\mathrm{M}$, et al. Telomere length changes during critical illness: a prospective, observational study. Genes 2019;10:761.

14 Carfi A, Bernabei R, Landi F, et al. Persistent symptoms in patients after acute COVID-19. JAMA 2020:324:603-5. 